\title{
Heparin in COVID-19 Patients Is Associated with Reduced In-Hospital Mortality: The Multicenter Italian CORIST Study*
}

Augusto Di Castelnuovo ${ }^{1, * *}$ Simona Costanzo ${ }^{2, * *(1)}$ Andrea Antinori ${ }^{3} \quad$ Nausicaa Berselli ${ }^{4}$ Lorenzo Blandi ${ }^{5}$ Marialaura Bonaccio ${ }^{2}$ Roberto Cauda ${ }^{6,7}$ Giovanni Guaraldi ${ }^{8}$ Lorenzo Menicanti ${ }^{9}$ Marco Mennuni ${ }^{10}$ Giustino Parruti ${ }^{11}$ Giuseppe Patti ${ }^{10}$ Francesca Santilli ${ }^{12}$ Carlo Signorelli ${ }^{13}$ Alessandra Vergori ${ }^{14}$ Pasquale Abete ${ }^{15}$ Walter Ageno ${ }^{16}$ Antonella Agodi ${ }^{17}$ Piergiuseppe Agostoni ${ }^{18,19}$ Luca Aiello 20 Samir Al Moghazi ${ }^{21}$ Rosa Arboretti ${ }^{22}$ Marinella Astuto ${ }^{23}$ Filippo Aucella ${ }^{24}$ Greta Barbieri ${ }^{25}$ Alessandro Bartoloni ${ }^{26}$ Paolo Bonfanti ${ }^{27,28}$ Francesco Cacciatore ${ }^{15}$ Lucia Caiano ${ }^{16}$ Laura Carrozzi $^{29}$ Antonio Cascio ${ }^{30}$ Arturo Ciccullo ${ }^{6}$ Antonella Cingolani6,7 Francesco Cipollone ${ }^{12}$ Claudia Colomba $^{30}$ Crizia Colombo $^{10}$ Francesca Crosta ${ }^{11}$ Gian Battista Danzi ${ }^{31}$ Damiano D’Ardes ${ }^{12}$ Katleen de Gaetano Donati ${ }^{6}$ Francesco Di Gennaro ${ }^{32}$ Giuseppe Di Tano ${ }^{31}$ Gianpiero D’Offizi ${ }^{33}$ Massimo Fantoni 6,7 Francesco Maria Fusco ${ }^{34}$ Ivan Gentile ${ }^{35}$ Francesco Gianfagna ${ }^{1,16}$ Elvira Grandone 24 Emauele Graziani ${ }^{36}$ Leonardo Grisafi ${ }^{10}$ Gabriella Guarnieri ${ }^{37}$ Giovanni Larizza ${ }^{38}$ Armando Leone ${ }^{39}$ Gloria Maccagni ${ }^{31}$ Ferruccio Madaro ${ }^{38}$ Stefano Maitan ${ }^{20}$ Sandro Mancarella ${ }^{40}$ Massimo Mapelli ${ }^{18,19}$ Riccardo Maragna ${ }^{18,19}$ Rossella Marcucci ${ }^{26}$ Giulio Maresca ${ }^{41}$ Silvia Marongiu ${ }^{42}$ Claudia Marotta $^{32}$ Lorenzo Marra $^{39}$ Franco Mastroianni ${ }^{38}$ Maria Mazzitelli ${ }^{43}$ Alessandro Mengozzi ${ }^{44}$ Francesco Menichetti ${ }^{44}$ Marianna Meschiari ${ }^{8}$ Jovana Milic ${ }^{8}$ Filippo Minutolo ${ }^{45}$ Beatrice Molena ${ }^{37}$ Arturo Montineri ${ }^{46}$ Cristina Mussini ${ }^{8}$ Maria Musso ${ }^{47}$ Daniela Niola ${ }^{12}$ Anna Odone ${ }^{5}$ Marco Olivieri ${ }^{48}$ Antonella Palimodde ${ }^{42}$ Roberta Parisi ${ }^{2}$ Emanuela Pasi ${ }^{36}$ Raffaele Pesavento ${ }^{49}$ Francesco Petri ${ }^{27}$ Biagio Pinchera ${ }^{35}$ Venerino Poletti ${ }^{50,51}$ Claudia Ravaglia ${ }^{50}$ Andrea Rognoni ${ }^{10}$ Marco Rossato ${ }^{49}$ Marianna Rossi ${ }^{27}$ Vincenzo Sangiovanni ${ }^{34}$ Carlo Sanrocco $^{11}$ Laura Scorzolini ${ }^{52}$ Raffaella Sgariglia ${ }^{40}$ Paola Giustina Simeone ${ }^{11}$ Eleonora Taddei ${ }^{6}$ Carlo Torti ${ }^{43}$ Roberto Vettor ${ }^{49}$ Andrea Vianello ${ }^{37}$ Marco Vinceti ${ }^{4,53}$ Alexandra Virano ${ }^{16}$ Laura Vocciante ${ }^{41}$ Raffaele De Caterina ${ }^{29}$ Licia lacoviello2,16

Thromb Haemost 2021;121:1054-1065.

${ }^{1}$ Mediterranea Cardiocentro, Napoli, Italy

2 Department of Epidemiology and Prevention, IRCCS Neuromed, Pozzilli, Isernia, Italy

3 UOC Immunodeficienze Virali, National Institute for Infectious Diseases “L. Spallanzani," IRCCS, Rome, Italy

4 Section of Public Health, Department of Biomedical, Metabolic and Neural Sciences, University of Modena and Reggio Emilia, Modena, Italy
Address for correspondence Licia lacoviello, MD, PhD, Department of Epidemiology and Prevention, IRCCS Neuromed, Via dell'Elettronica, 86077 Pozzilli, Isernia, Italy (e-mail: licia.iacoviello@moli-sani.org).

\footnotetext{
5 Università di Pavia, Pavia, Italy

${ }^{6}$ Fondazione Policlinico Universitario A, Gemelli IRCCS, Rome, Italy

7 Dipartimento di Sicurezza e Bioetica Sede di Roma, Università Cattolica del Sacro Cuore, Rome, Italy

8 Infectious Disease Unit, Department of Surgical, Medical, Dental and Morphological Sciences, University of Modena and Reggio Emilia, Modena, Italy
}

\footnotetext{
* THE COVID-19 RISK and Treatments (CORIST) collaboration.

${ }^{* *}$ These authors contributed equally to this work.
}

received

December 14, 2020

accepted after revision

January 6, 2021

published online

January 7, 2021 (c) 2021. Thieme. All rights reserved. Georg Thieme Verlag KG,

Rüdigerstraße 14,

70469 Stuttgart, Germany
DOI https://doi.org/ 10.1055/a-1347-6070. ISSN 0340-6245. 
${ }^{9}$ IRCCS Policlinico San Donato, San Donato, Milanese (MI), Italy

10 University of Eastern Piedmont, Maggiore della Carità Hospital, Novara, Italy

${ }^{11}$ Department of Infectious Disease, Azienda Sanitaria Locale (AUSL) di Pescara, Pescara, Italy

${ }^{12}$ Department of Medicine and Aging, Clinica Medica, "SS. Annunziata" Hospital and University of Chieti, Chieti, Italy

13 School of Medicine, Vita-Salute San Raffaele University, Milan, Italy

${ }^{14}$ Department of HIV/AIDS, National Institute for Infectious Diseases “Lazzaro Spallanzani”-IRCCS, Rome, Italy

${ }^{15}$ Dipartimento di Scienze Mediche Traslazionali, Università degli studi di Napoli “Federico II," Napoli, Italy

16 Department of Medicine and Surgery, University of Insubria, Varese, Italy

17 Department of Medical and Surgical Sciences and Advanced Technologies "G.F. Ingrassia," University of Catania; AOU Policlinico "G. Rodolico - San Marco," Catania, Italy

${ }^{18}$ Centro Cardiologico Monzino IRCCS, Milan, Italy

${ }^{19}$ Section of Cardiovascular, Department of Clinical Sciences and Community Health, University of Milano, Milan, Italy

20 UOC, Anestesia e Rianimazione, Dipartimento di Chirurgia Generale Ospedale Morgagni-Pierantoni, Forlì, Italy

${ }^{21}$ UOC Infezioni Sistemiche dell'Immunodepresso, National Institute for Infectious Diseases L. Spallanzani, IRCCS, Rome, Italy

22 Department of Civil Environmental and Architectural Engineering, University of Padova, Padova, Italy

${ }^{23}$ Department of General Surgery and Medical-Surgical Specialties, University of Catania, U.O. Anestesia, Rianimazione 1. P.O. "G. Rodolico," AOU Policlinico "G. Rodolico - San Marco," Catania, Italy

${ }^{24}$ Fondazione I.R.C.C.S "Casa Sollievo della Sofferenza," San Giovanni Rotondo, Foggia, Italy

25 Department of Surgical, Medical and Molecular Medicine and Critical Care, Azienda Ospedaliera Universitaria Pisana, University of Pisa, Pisa, Italy

26 Department of Experimental and Clinical Medicine, University of Florence and Azienda Ospedaliero-Universitaria Careggi, Firenze, Italy

${ }^{27}$ UOC Malattie Infettive, Ospedale San Gerardo, ASST Monza, Monza, Italy

${ }^{28}$ School of Medicine and Surgery, University of Milano-Bicocca, Milan, Italy

${ }^{29}$ Cardiovascular and Thoracic Department, Azienda OspedalieroUniversitaria Pisana and University of Pisa, Pisa, Italy
30 Infectious and Tropical Diseases Unit, Department of Health Promotion, Mother and Child Care, Internal Medicine and Medical Specialties (PROMISE), University of Palermo, Palermo, Italy

31 Department of Cardiology, Ospedale di Cremona, Cremona, Italy

32 Medical Direction, IRCCS Neuromed, Pozzilli, Isernia, Italy

${ }^{3}$ UOC Malattie Infettive-Epatologia, National Institute for Infectious Diseases L, Spallanzani, IRCCS, Roma, Italy

${ }^{34}$ UOC Infezioni Sistemiche e dell'Immunodepresso, Azienda Ospedaliera dei Colli, Ospedale Cotugno, Napoli, Italy

${ }^{35}$ Department of Clinical Medicine and Surgery, University of Naples Federico II, Napoli, Italy

36 Medicina Interna, Ospedale di Ravenna, AUSL della Romagna, Ravenna, Italy

${ }^{37}$ Division of Respiratory Pathophysiology, Department of Cardiologic, Thoracic and Vascular Sciences, University of Padova, Padova, Italy

${ }^{38}$ COVID-19 Unit, EE Ospedale Regionale F. Miulli, Acquaviva delle Fonti, Bari, Italy

${ }^{39}$ UOC di Pneumologia, P.O. San Giuseppe Moscati, Taranto, Italy

40 ASST Milano Nord, Ospedale Edoardo Bassini Cinisello Balsamo, Milan, Italy

41 UOC di Medicina - Presidio Ospedaliero S. Maria di Loreto Nuovo. ASL Napoli 1 Centro. Napoli. Italy

42 P.O. Santissima Trinità di Cagliari, Cagliari, Italy

43 Infectious and Tropical Diseases Unit, Department of Medical and Surgical Sciences "Magna Graecia" University, Catanzaro, Italy

${ }^{44}$ Department of Clinical and Experimental Medicine, Azienda Ospedaliera Universitaria Pisana, University of Pisa, Pisa, Italy

${ }^{45}$ Dipartimento di Farmacia, Università di Pisa, Pisa, Italy

46 U.O. C. Malattie Infettive e Tropicali, P.O. "San Marco." AOU Policlinico "G. Rodolico - San Marco," Catania, Italy

47 UOC Malattie Infettive-Apparato Respiratorio, National Institute for Infectious Diseases “L. Spallanzani," IRCCS, Rome, Italy

${ }^{48}$ Computer Service, University of Molise, Campobasso, Italy

${ }^{49}$ Clinica Medica 3, Department of Medicine - DIMED, University hospital of Padova, Padova, Italy

50 UOC Pneumologia, Dipartimento di Malattie Apparato Respiratorio e Torace, Ospedale Morgagni-Pierantoni, Forlì, Italy

51 Department of Respiratory Diseases and Allergy, Aarhus University Hospital, Aarhus, Denmark

${ }^{52}$ UOC Malattie Infettive ad Alta Intensità di Cura, National Institute for Infectious Diseases “L. Spallanzani,” IRCCS, Rome, Italy

${ }^{53}$ Department of Epidemiology, Boston University School of Public Health, Boston, Massachusetts, United States

\section{Abstract}

Introduction A hypercoagulable condition was described in patients with coronavirus disease 2019 (COVID-19) and proposed as a possible pathogenic mechanism contributing to disease progression and lethality.

Aim We evaluated if in-hospital administration of heparin improved survival in a large cohort of Italian COVID-19 patients.

Methods In a retrospective observational study, 2,574 unselected patients hospitalized in 30 clinical centers in Italy from February 19, 2020 to June 5, 2020 with laboratory-confirmed severe acute respiratory syndrome coronavirus- 2 infection were analyzed. The primary endpoint in a time-to event analysis was in-hospital death, comparing patients who received heparin (low-molecular-weight heparin [LMWH] or unfractionated heparin [UFH]) with patients who did not. We used multivariable Cox proportional-hazards regression models with inverse probability for treatment weighting by propensity scores. 

Keywords
- COVID-19
- coronavirus
- heparin
- coagulation activation
- treatments
- mortality

Results Out of 2,574 COVID-19 patients, 70.1\% received heparin. LMWH was largely the most used formulation (99.5\%). Death rates for patients receiving heparin or not were 7.4 and 14.0 per 1,000 person-days, respectively. After adjustment for propensity scores, we found a $40 \%$ lower risk of death in patients receiving heparin (hazard ratio $=0.60$; $95 \%$ confidence interval: $0.49-0.74$; E-value $=2.04$ ). This association was particularly evident in patients with a higher severity of disease or strong coagulation activation.

Conclusion In-hospital heparin treatment was associated with a lower mortality, particularly in severely ill COVID-19 patients and in those with strong coagulation activation. The results from randomized clinical trials are eagerly awaited to provide clear-cut recommendations.

\section{Introduction}

Coronavirus disease 2019 (COVID-19), provoked by severe acute respiratory syndrome coronavirus-2 (SARS-CoV-2) infection, has afflicted millions of people globally with an impressive cost in death and disabilities that is still rapidly rising around the world. Awaiting for the development of effective and safe vaccines and specific antiviral therapies, researchers are struggling to better understand the disease and optimize supportive treatment. A hypercoagulable condition, associated with the presence of microvascular thrombosis, has been described in patients with COVID-19 ${ }^{1-5}$ and proposed as a possible pathogenic mechanism contributing to disease progression and lethality. Therefore, the possibility to improve clinical outcomes of COVID-19 patients by commonly used and inexpensive antithrombotic drugs has become attractive, although data on the risk-benefit profile of anticoagulant (AC) therapy in COVID-19 have been sparse and contradictory.

Recent data show that treatment by low-molecular-weight heparin (LMWH) or unfractionated heparin (UFH) at prophylactic doses is associated with reduced 28-day mortality in severe COVID-19 patients featuring a high level of activation of the coagulation system. ${ }^{6}$ Moreover, in a large cohort of COVID19-hospitalized patients, treatment doses of AC have been reported to be associated with improved outcomes. ${ }^{7}$ Other studies, however, have been unable to find any efficacy of prediagnosis anticoagulation among either hospitalized or ambulatory patients with COVID-19 infection. ${ }^{8}$ It is still not clear if pulmonary vessel occlusions observed in COVID-19 patients derive from embolism from peripheral vein thrombosis $^{9}$ or from locally generated thrombi. ${ }^{10-12}$ The pathogenic substrate of pulmonary occlusions implies different AC therapeutic approaches, with either therapeutic or prophylactic doses of heparin or the use of other antithrombotic therapies, ${ }^{13}$ an issue still unsolved by available studies. Differing recommendations by guidelines on thromboprophylaxis and $\mathrm{AC}$ therapy in COVID-19 have thus been issued. ${ }^{14-20}$ The World Health Organization, the U.S. Center for Disease Control and Prevention and the Department of Defense have recommended the use of prophylactic doses of heparin or LMWH for prevention of venous thromboembolism (VTE) in hospitalized adults and adolescents with severe COVID-19 disease, unless contraindicated. ${ }^{14-16}$ The Italian Society on Thrombosis and Haemostasis $^{17}$ and a recent position paper endorsed by several international societies ${ }^{18,19}$ have suggested a VTE risk stratification for all individuals with COVID-19 and extended postdischarge thromboprophylaxis in patients at a higher risk of VTE, recognizing insufficient evidence to recommend the empiric use of therapeutic doses of UFH or LMWH. Other agencies have suggested intermediate or therapeutic doses of LMWH for hospitalized patients and extended VTE prophylaxis for up to 45 days postdischarge. ${ }^{18}$ Recently, the VAS-European Independent Foundation in Angiology/Vascular Medicine drew attention to patients with vascular disease and COVID-19, recommending LMWH (weight-adjusted intermediate doses, unless contraindicated) as the drug of choice over UFH or direct oral ACs for the treatment of VTE or hypercoagulability. ${ }^{20} \mathrm{Hard}$ data on the efficacy of heparin treatment either at therapeutic or at prophylactic doses are not conclusive.

We therefore evaluated the association between in-hospital administration of heparin and survival in a large cohort of patients with COVID-19 hospitalized in 30 centers all over Italy.

\section{Methods}

\section{Setting}

This national retrospective observational study was conceived, coordinated, and analyzed within the CORIST project (ClinicalTrials.gov ID: NCT04318418). ${ }^{21-23}$ The study was approved by the institutional ethics board of the Istituto di Ricovero e Cura a Carattere Scientifico (IRCCS) Neuromed, Pozzilli, and of all recruiting centers. Each hospital provided data from hospitalized patients who had a positive test result for the SARS-CoV-2 virus at any time during their hospitalization from February 19 to June 5, 2020. The follow-up continued through June 30, 2020.

The acquisition of retrospective information about inhospital heparin use started in April 2020, and 30 centers (listed in the -Supplementary Material, available in the online version) provided data. The acceptance to participate in the project or to provide data for the present analysis was not related to the use of heparin. 


\section{Data Sources}

In the framework of the CORIST study, we developed a cohort comprising 4,312 patients with laboratory-confirmed SARS$\mathrm{CoV}-2$ infection in an in-patient setting. The SARS-CoV-2 status was declared based on laboratory results (polymerase chain reaction on a nasopharyngeal swab) from each participating hospital. Clinical data were abstracted at one-time point from electronic medical records or charts, and collected using either a centrally designed electronic worksheet or a centralized web-based database. Collected data included patients' demographics, laboratory test results, medication administration, historical and current medication lists, historical and current diagnoses, and clinical notes. In addition, specific information on the most severe manifestations of COVID-19 occurred during hospitalization was retrospectively captured. Following the World Health Organization criteria, the maximum clinical severity observed was classified as mild pneumonia (pneumonia and no signs of severe pneumonia); or severe pneumonia (fever or suspected respiratory infection, plus one of respiratory rates $>30$ breaths/min, severe respiratory distress, or $\mathrm{SpO}_{2}<90 \%$ on room air); or acute respiratory distress syndrome. ${ }^{24}$ Specifically, we obtained the following information for each patient: hospital; date of admission and date of discharge or death; age; sex; the first recorded inpatient laboratory tests at admission (creatinine, C-reactive protein, D-dimer); past and current diagnoses (myocardial infarction, heart failure, diabetes, hypertension, respiratory disease, and cancer), and current drug therapies for COVID-19 (heparin, hydroxychloroquine [HCQ], lopinavir/ritonavir or darunavir/cobicistat, remdesivir, tocilizumab or sarilumab, and corticosteroids). A diagnosis of pre-existing cardiovascular disease was based on a history of myocardial infarction or heart failure. Diagnosis of chronic kidney disease was based on creatinine values at entry and classified as: stage 1: kidney damage with normal or increased glomerular filtration rate (GFR; $>90 \mathrm{~mL} / \mathrm{min} / 1.73 \mathrm{~m}^{2}$ ); stage 2 : mild reduction in GFR (60-89 $\left.\mathrm{mL} / \mathrm{min} / 1.73 \mathrm{~m}^{2}\right)$; stage 3a: moderate reduction in GFR (45-59 $\mathrm{mL} / \mathrm{min} / 1.73 \mathrm{~m}^{2}$ ); stage 3b: moderate reduction in GFR (30-44 mL/min/1.73 $\left.\mathrm{m}^{2}\right)$; stage 4 : severe reduction in GFR $\left(15-29 \mathrm{~mL} / \mathrm{min} / 1.73 \mathrm{~m}^{2}\right)$; stage 5 : kidney failure (GFR $<15$ $\mathrm{mL} / \mathrm{min} / 1.73 \mathrm{~m}^{2}$ or dialysis). For statistical analysis, stages 3a and $3 \mathrm{~b}$ and stages 4 and 5 were combined. GFR was calculated by the Chronic Kidney Disease Epidemiology Collaboration (CKD-EPI) equation.

Patients were defined as receiving heparin if they were receiving it at hospital admission or during the follow-up period. Every physician in each hospital decided for himself or herself if and how to treat their patient, including allocation to prophylaxis or therapeutic regimen. This last decision was based on existing Italian guidelines for deep venous thrombosis and pulmonary embolism prevention and therapy, schematized in - Supplementary Table $\mathbf{S 1}$ (available in the online version). Briefly, these guidelines suggest to allocate each patient, for whom heparin treatment was decided, to a prophylactic or therapeutic regimen based on body weight and GFR. We requested to each center the following information: (1) use of heparin during hospitalization (yes/no); (2) type (LMWH, UFH, etc.) and dosage or regimen (prophylactic or therapeutic). Information on type of heparin used and dosage or regimen was missing for $n=403$ patients; as a consequence, it was not possible to classify these patients as at prophylactic or therapeutic regimen. They were included in the main analyses but excluded from those by regimen. Additionally, we found $n=39$ patients treated with LMWH at the dose of 6,000 $\mathrm{IU} /$ day and missing data on body weight and regimen. These individuals were considered as at therapeutic regimen; however, such individuals if having body weight $>100 \mathrm{~kg}$ and GFR $\geq 30 \mathrm{~mL} / \mathrm{min}$ would have been considered as at prophylactic instead of therapeutic regimen. So, we conducted a sensitivity analysis shifting these individuals in the prophylactic group or excluding them from analyses by regimen. Heparin was used in the early phase after hospitalization, with or without previous laboratory evidence of coagulation activation at entry. Precise data at individual level concerning when heparin treatment was started and how long heparin was given for each patient were not available.

\section{Statistical Analysis}

The study index date was defined as the date of hospital admission. Index dates ranged from February 19, 2020 to June 5, 2020. The study endpoint was the time from study index date to death. The number of patients who either died, had been discharged alive, or were still hospitalized as of June 30, 2020 were recorded, and hospital duration of stay was determined. Patients alive had their data censored on the date of discharge or as the date of the respective clinical data collection. Data were censored at 35 days of follow-up in $n=430(10.0 \%)$ patients with a follow-up longer than 35 days.

Since gathering of information on heparin use started almost 1 month after the CORIST project opening, data on in-hospital heparin use, as anti-COVID-19 treatment, were available for 2,723 patients only. Of them, 149 patients died or were discharged within 24 hours after admission to the hospital, and were thus excluded from the analysis. - Supplementary Table S2 (available in the online version) shows the comparisons between the entire cohort (with or without information on heparin use) and the final sample used for the present analysis. No difference was found for the prevalence of age, gender, chronic degenerative diseases, and mortality rate.

Among 2,574 COVID-19 patients, 245 (9.5\%) had at least one missing value for covariates. Distribution of missing values was as follows: $n=137$ for C-reactive protein; $n=64$ for GFR; $n=74$ for history of ischemic disease; $n=62$ for history of chronic pulmonary disease; $n=49$ for diabetes; $n=49$ for hypertension; and $n=53$ for cancer. We used multiple imputation techniques (SAS PROC MI, $n=10$ imputed datasets; and PROC MIANALYZE) to maximize data availability. As sensitivity analysis, we also conducted a casecomplete analysis on 2,329 patients.

We used Cox proportional-hazards regression models to estimate the association between heparin use and death. Since we applied multiple imputation, we obtained the final standard error using the Rubin's rule based on the robust variance estimator in Cox regression. ${ }^{25}$ We assessed the 
proportional hazards assumption using weighted Schoenfeld residuals, and identified no violations. To account for the nonrandomized heparin administration and to reduce the effects of confounding, we used the propensity score method. We assessed the individual propensities for receiving heparin treatment with the use of a multivariable logistic-regression model that included age, sex, diabetes, hypertension, history of ischemic heart disease, chronic pulmonary disease, GFR, C-reactive protein, hospital clustering, and use of other drug therapies for COVID-19 (HCQ, lopinavir/ritonavir or darunavir/cobicistat, remdesivir, corticosteroids, tocilizumab, or sarilumab). We then appraised associations between heparin treatment and death by multivariable Cox regression models with the use of propensity score, and further controlling for hospital clustering as a random effect (frailty model). We chose the use of a frailty model as suggested by Glidden and Vittinghoff. ${ }^{26}$ The primary analysis used inverse probability by treatment weighting; we used the predicted probabilities from the propensity-score model to calculate the stabilized inverse-probability-weighting weight. ${ }^{27}$ Stabilized weights were normalized so that they added up the actual sample size. Secondary analyses used propensityscore stratification ( $n=5$ strata) or multivariable Cox regression analysis or multivariable logistic regression analyses comparing dead patients versus alive patients, or accounted for hospital clustering via stratification or by a robust sandwich estimator. We conducted pre-established subgroup analyses according to age and sex of patients, degree of COVID-19 severity experienced during the hospital stay, Creactive protein at baseline, CKD stage, stay in intensive care unit (ICU), and other drug therapies for COVID-19. Additionally, we investigated the association of different regimens (prophylactic or therapeutic doses) with the risk of inhospital mortality. We performed a further sensitivity analysis according to quartiles of $\mathrm{D}$-dimer in a subsample of 1,792 COVID-19 patients with nonmissing data on D-dimer.

We clustered hospitals according to their geographical distribution: hospitals from northern regions of Italy, Milan excluded $(n=793)$, Milan $(n=189)$; hospitals from central regions of Italy, Rome excluded ( $n=1,003)$, Rome $(n=254)$; hospitals from southern regions of Italy $(n=335)$; see the list of centers in the Supplementary Material (available in the online version). To quantify the potential for an unmeasured confounder to render apparent statistically significant hazard ratios (HRs) nonsignificant, we calculated the E-value. ${ }^{28}$ We performed all analyses with the aid of the SAS version 9.4 statistical software for Windows.

\section{Results}

In the final current analyses, we included 2,574 patients who were hospitalized with confirmed SARS-CoV-2 infection at 30 clinical centers across Italy who either died or had been discharged or were still in hospital as of June 30,2020 . Of these patients, 1,804 (70.1\%) received heparin during hospitalization. The information on type of heparin used was missing for $n=403$ out of 1,804 . In the others, LMWH and UFH were the types used in 99.5 and $0.5 \%$, respectively.
Baseline characteristics according to heparin use are shown in - Table 1. Patients receiving heparin were more likely older, had higher levels of C-reactive protein at entry, and showed higher prevalence of diabetes, hypertension, chronic pulmonary disease and cancer, but a lower prevalence of ischemic heart disease (-Table 1). Patients in the heparin group had a higher degree of COVID-19 severity and were more likely to receive another drug for COVID-19 treatment (75.5\%; lopinavir/ritonavir or darunavir/cobicistat, remdesivir, tocilizumab or sarilumab, corticosteroids, or $83.7 \% \mathrm{HCQ}$ ), in comparison with nonheparin-receiving patients $(p<0.0001 ;-$ Table 1$)$. D-dimer levels were not associated with heparin therapy.

The unadjusted differences and differences adjusted by propensity scores between heparin-treated and nonheparintreated patients for each variable included in the propensity score are shown in - Supplementary Fig. $\mathbf{5 1}$ (available in the online version). All pretreatment differences disappeared after adjustment by propensity score weighting. The Cstatistic of the propensity-score model was 0.78 .

\section{Primary Outcome}

Out of 2,574 patients, 398 died (15.5\%), 1,687 were discharged alive (65.5\%), and 489 (19.0\%) were still at the hospital. The median follow-up was 15 days (interquartile range: 9-25; range: $2-35 ; 43,773$ person-days). The death rate (per 1,000 person-days) was 7.4 in heparin- and 14.0 in nonheparintreated patients ( - Table 2 ). At univariable analysis, HR for mortality was 0.54 (95\% confidence interval [CI]: 0.44-0.67). In the primary multivariable analysis with inverse probability weighting according to the propensity score, heparin use was associated with a $40 \%$ (95\% CI: $26-51 \%)$ lower risk of death $(-$ Fig. 1 and $\sim$ Table 2; E-value $=2.04$ ).

Secondary multivariable analyses, as well as casecomplete analyses restricted to 2,329 patients without missing data, yielded very similar results $(-$ Table 2$)$. Considering secondary multivariable analyses overall, HR for mortality associated with heparin ranged between 0.53 and 0.62 according to the type of analysis. Control of hospital clustering with different approaches also yielded similar results for the primary analysis ( $\mathbf{- S u p p l e m e n t a r y ~ T a b l e ~} \mathbf{5 3}$ [available in the online version]). As an additional sensitivity analysis, we present in -Supplementary Table $\mathbf{5 4}$ (available in the online version) the main findings after exclusion of $n=403$ patients for whom the type of heparin used was missing. Heparin used remains clearly associated with a lower mortality ( $\mathrm{HR}=0.52$; 95\% CI: $0.41-0.65)$.

We present subgroup analyses in - Table 3 . Heparin use remained consistently associated with a lower mortality in almost all subgroups. The inverse association of heparin use with in-hospital mortality was more evident in patients who experienced a higher degree of COVID-19 severity or were staying in the ICU.

Stratification of patients by the number of in-hospital anti-COVID-19 treatments showed that heparin was more effective when other drugs were also used ( - Table 3 ).

Among patients treated with heparin, $54.5 \%$ were at prophylactic and $23.2 \%$ at the therapeutic regimen, while 
Table 1 General characteristics of COVID-19 patients at baseline, according to heparin use

\begin{tabular}{|c|c|c|c|}
\hline \multirow[t]{2}{*}{ Characteristic } & \multicolumn{2}{|l|}{ Heparin } & \multirow[t]{2}{*}{$p$-Value unadjusted } \\
\hline & No $(N=770)$ & Yes $(N=1,804)$ & \\
\hline Age (y), median (IQR) & $65(53-77)$ & $68(57-79)$ & $<0.0001$ \\
\hline Gender, no. (\%) & & & 0.064 \\
\hline Women & $275(35.7)$ & $714(39.6)$ & \\
\hline Men & $495(64.3)$ & $1,090(60.4)$ & \\
\hline Diabetes, no. (\%) & & & $<0.0001$ \\
\hline No & $623(80.9)$ & $1,394(77.3)$ & \\
\hline Yes & $121(15.7)$ & $387(21.4)$ & \\
\hline Missing data & $26(3.4)$ & $23(1.3)$ & \\
\hline Hypertension, no. (\%) & & & $<0.0001$ \\
\hline No & $390(50.7)$ & $841(46.6)$ & \\
\hline Yes & $351(45.6)$ & $943(52.3)$ & \\
\hline Missing data & $29(3.8)$ & $20(1.1)$ & \\
\hline Ischemic heart disease, no. (\%) & & & 0.0074 \\
\hline No & $587(76.2)$ & $1,465(81.2)$ & \\
\hline Yes & $152(19.8)$ & $296(16.4)$ & \\
\hline Missing data & $31(4.0)$ & $43(2.4)$ & \\
\hline Chronic pulmonary disease, no. (\%) & & & $<0.0001$ \\
\hline No & $656(85.2)$ & $1,480(82.0)$ & \\
\hline Yes & $82(10.6)$ & $294(16.3)$ & \\
\hline Missing data & $32(4.2)$ & $30(1.7)$ & \\
\hline Cancer, no. (\%) & & & $<0.0001$ \\
\hline No & $678(88.1)$ & $1,563(86.6)$ & \\
\hline Yes & $63(8.2)$ & $217(12.0)$ & \\
\hline Missing data & $29(3.8)$ & $24(1.3)$ & \\
\hline \multicolumn{3}{|c|}{ Highest degree of COVID-19 severity experienced at hospital, no. (\%) } & $<0.0001$ \\
\hline Mild pneumonia & $431(56.0)$ & $790(43.8)$ & \\
\hline Severe pneumonia & $227(29.5)$ & $509(28.2)$ & \\
\hline Acute respiratory distress syndrome & $112(14.5)$ & $505(28.0)$ & \\
\hline ICU, no. (\%) & & & $<0.0001$ \\
\hline No & $663(86.1)$ & $1,517(84.1)$ & \\
\hline Yes & $65(8.4)$ & $262(14.5)$ & \\
\hline Missing data & $42(5.5)$ & $25(1.4)$ & \\
\hline CKD stage ${ }^{a}$, no. (\%) & & & $<0.0001$ \\
\hline Stage 1 & $286(37.2)$ & $592(32.8)$ & \\
\hline Stage 2 & $278(36.1)$ & $693(38.4)$ & \\
\hline Stage 3a or stage 3b & $135(17.5)$ & $355(19.7)$ & \\
\hline Stage 4 or stage 5 & $37(4.8)$ & $134(7.4)$ & \\
\hline Missing data & $34(4.4)$ & $30(1.7)$ & \\
\hline C-reactive protein, no. (\%) & & & $<0.0001$ \\
\hline$<1 \mathrm{mg} / \mathrm{L}$ & $90(11.7)$ & $155(8.6)$ & \\
\hline $1-3 \mathrm{mg} / \mathrm{L}$ & $108(14.3)$ & $142(7.9)$ & \\
\hline$>3 \mathrm{mg} / \mathrm{L}$ & $524(68.1)$ & $1,418(78.6)$ & \\
\hline
\end{tabular}


Table 1 (Continued)

\begin{tabular}{|c|c|c|c|}
\hline \multirow[t]{2}{*}{ Characteristic } & \multicolumn{2}{|l|}{ Heparin } & \multirow[t]{2}{*}{$p$-Value unadjusted } \\
\hline & No $(N=770)$ & Yes $(N=1,804)$ & \\
\hline Missing data & $48(6.2)$ & $89(4.9)$ & \\
\hline \multicolumn{2}{|c|}{ In-hospital therapies for COVID-19, no. (\%) } & & $<0.0001$ \\
\hline No & $269(34.9)$ & $441(24.5)$ & \\
\hline Yes & $501(65.1)$ & $1,363(75.5)$ & \\
\hline Hydroxychloroquine, no. (\%) & & & $<0.0001$ \\
\hline No & $336(43.6)$ & $295(16.4)$ & \\
\hline Yes & $434(56.4)$ & $1,509(83.7)$ & \\
\hline Subsample, $N=1,792$ & No $(N=369)$ & Yes $(N=1,423)$ & \\
\hline D-dimer, quartiles, no. (\%) & & & 0.13 \\
\hline IQ: $\leq 470 \mathrm{ng} / \mathrm{mL}$ & $104(28.2)$ & $346(24.3)$ & \\
\hline IIQ: $471-899 \mathrm{ng} / \mathrm{mL}$ & $76(20.6)$ & $361(25.4)$ & \\
\hline IIIQ: $900-2,020 \mathrm{ng} / \mathrm{mL}$ & $102(27.6)$ & $355(25.0)$ & \\
\hline IVQ: > 2,020 ng/mL & 87 (23.6) & $361(25.4)$ & \\
\hline
\end{tabular}

Abbreviations: CKD, chronic kidney disease; ICU, intensive care unit.

aStage 1: kidney damage with normal or increased glomerular filtration rate (GFR) (>90 mL/min/1.73 $\left.\mathrm{m}^{2}\right)$; stage 2: mild reduction in GFR $\left(60-89 \mathrm{~mL} / \mathrm{min} / 1.73 \mathrm{~m}^{2}\right)$; stage 3a: moderate reduction in GFR $\left(45-59 \mathrm{~mL} / \mathrm{min} / 1.73 \mathrm{~m}^{2}\right)$; stage 3b: moderate reduction in GFR $(30-44 \mathrm{~mL} / \mathrm{min} /$ $\left.1.73 \mathrm{~m}^{2}\right)$; stage 4: severe reduction in GFR $\left(15-29 \mathrm{~mL} / \mathrm{min} / 1.73 \mathrm{~m}^{2}\right)$; stage 5 : kidney failure (GFR $<15 \mathrm{~mL} / \mathrm{min} / 1.73 \mathrm{~m}{ }^{2}$ or dialysis).

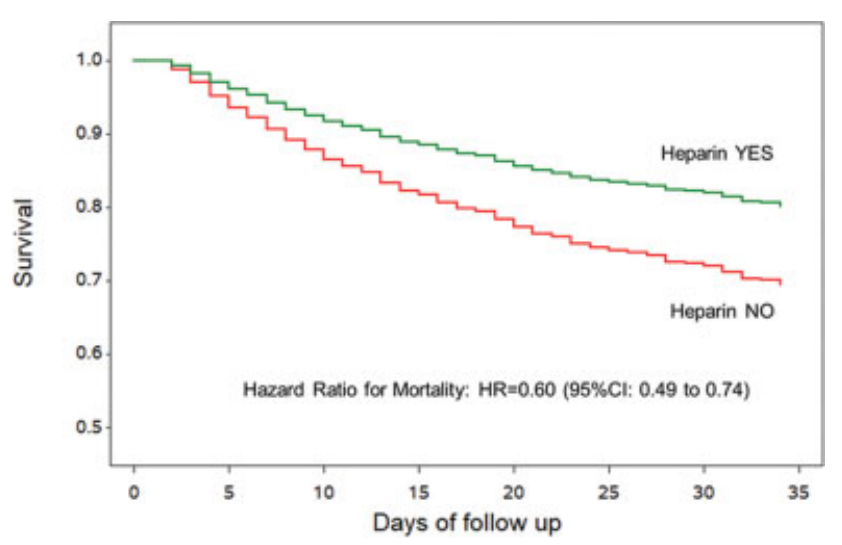

Fig. 1 Survival curves according to heparin use. The curves are adjusted by propensity score analysis (inverse probability weighting) and age and sex as fixed effects and hospital index as random effect, and are generated using the first imputed dataset. The other imputed datasets are similar and thus omitted.

for the remaining $22.3 \%$ it was not possible to proceed with this classification because of missing data. In comparison with no heparin use, treatment at prophylactic and therapeutic doses was associated with a 60 and $35 \%$ lower relative risk of mortality, respectively (-Table 4). Thirty-nine patients were treated with LMWH at the dose of 6,000 IU/day and they have been considered in the previous analysis as at the therapeutic regimen (see the Methods section for clarification). At sensitivity analysis, we shifted these patients to the prophylactic regimen or excluded them from the analysis, and the findings in - Table $\mathbf{4}$ were very similar (data not shown).

At the end, in the analysis restricted to 1,792 COVID-19 patients without missing data on D-dimer levels, heparin use was associated with a lower in-hospital mortality in patients in the highest quartiles (with D-dimer $>2,020.00 \mathrm{ng} / \mathrm{mL}$; $\mathrm{HR}=0.63$, 95\% CI: 0.41-0.97) (-Fig. 2).

\section{Discussion}

It is not yet clear if heparin use is an effective intervention in all hospitalized COVID-19 patients or should be limited to specific conditions or categories of patients and which doses should be used. ${ }^{17,18,20,29}$ We here show, in a large cohort of unselected COVID-19 patients hospitalized throughout Italy, that treatment with heparin (mainly LMWH) is associated with a lower risk of in-hospital mortality, independently from several possible confounders and taking into account possible differences across centers by either adjustment or stratification. To further minimize the possible bias due to the observational design, we used different analytical approaches aiming at creating an overall balance between comparison groups. Finally, we tried to limit the bias due to missing data by using a multiple imputation approach, but in no case, results were changed.

COVID-19 infection has been clinically divided into three phases: a first phase with unspecific symptoms; followed by a second phase with pulmonary impairment and inflammation activation; and a third phase, affecting only a limited number of patients, characterized by a worsening of clinical symptoms and a widespread inflammatory state. The last has been related to a process of "hypercoagulation" that could be potentially controlled by AC therapy. Therefore, heparin might be useful both in the initial phase as prophylactic treatment in patients with pneumonia and in advanced stages to contain the thrombotic phenomena that originate 
Table 2 Incidence rates and hazard ratios for death in COVID-19 patients according to heparin use

\begin{tabular}{|c|c|c|c|c|}
\hline \multicolumn{5}{|c|}{ Multiple imputation analysis $(\mathrm{N}=2,574)$} \\
\hline & Death $(N=398)$ & Patients at risk $(N=2,574)$ & Person-days & Death rate ( $x 1,000$ person-days) \\
\hline \multicolumn{5}{|l|}{ Heparin } \\
\hline No, no. (\%) & $145(18.8 \%)$ & $770(100 \%)$ & 10,362 & 14.0 \\
\hline Yes, no. (\%) & $253(14.0 \%)$ & $1,804(100 \%)$ & 34,301 & 7.4 \\
\hline \multicolumn{4}{|c|}{ Hazard ratio for mortality (heparin vs. nonheparin) } & $\mathrm{HR}(95 \% \mathrm{Cl})$ \\
\hline \multicolumn{4}{|c|}{ Crude analysis } & $0.54(0.44-0.67)$ \\
\hline \multicolumn{4}{|c|}{ Multivariable analysis $^{a}$} & $0.55(0.43-0.69)$ \\
\hline \multicolumn{4}{|c|}{ Propensity score analysis, inverse probability weighting ${ }^{\mathrm{b}}$ (main result) } & $0.60(0.49-0.74)$ \\
\hline \multicolumn{4}{|c|}{ Propensity score analysis, stratification $(n=5 \text { strata })^{\mathrm{b}}$} & $0.53(0.42-0.66)$ \\
\hline \multicolumn{4}{|c|}{ Odds ratio for mortality (heparin vs. no heparin) } & OR $(95 \% \mathrm{Cl})$ \\
\hline \multicolumn{4}{|c|}{ Multivariable analysis } & $0.53(0.40-0.71)$ \\
\hline \multicolumn{5}{|c|}{ Case complete analysis $(\mathrm{N}=2,329)$} \\
\hline & Death $(N=342)$ & Patients at risk $(N=2,329)$ & Person-days & Death rate ( $x 1,000$ person-days) \\
\hline \multicolumn{5}{|l|}{ Heparin } \\
\hline No, no. (\%) & $126(18.5 \%)$ & $682(100 \%)$ & 9,287 & 13.6 \\
\hline Yes, no. (\%) & $216(13.1 \%)$ & $1,647(100 \%)$ & 30,832 & 7.0 \\
\hline \multicolumn{4}{|c|}{ Hazard ratio for mortality (heparin vs. nonheparin) } & $\mathrm{HR}(95 \% \mathrm{Cl})$ \\
\hline \multicolumn{4}{|c|}{ Crude analysis } & $0.53(0.42-0.66)$ \\
\hline \multicolumn{4}{|c|}{ Multivariable analysis $^{\mathrm{a}}$} & $0.58(0.45-0.74)$ \\
\hline \multicolumn{4}{|c|}{ Propensity score analysis, inverse probability weighting ${ }^{\mathrm{b}}$} & $0.62(0.49-0.77)$ \\
\hline \multicolumn{4}{|c|}{ Propensity score analysis, stratification $(n=5 \text { strata })^{b}$} & $0.54(0.42-0.69)$ \\
\hline \multicolumn{4}{|c|}{ Odds ratio for mortality (heparin vs. no heparin) } & OR $(95 \% \mathrm{Cl})$ \\
\hline \multicolumn{4}{|c|}{ Multivariable analysis ${ }^{a}$} & $0.55(0.40-0.76)$ \\
\hline
\end{tabular}

Abbreviations: $\mathrm{Cl}$, confidence intervals; $\mathrm{HR}$, hazard ratios; OR, odds ratio.

${ }^{a}$ Controlling for age, sex, diabetes, hypertension, ischemic heart disease, chronic pulmonary disease, chronic kidney disease, C-reactive protein, HCQ, and other in-hospital therapies for COVID-19 as fixed effects and hospital index as a random effect.

bIncluding hospital index as a random effect covariate.

in the pulmonary circulation as a result of the worsening inflammatory state. Moreover, heparin, and in particular LMWH, may also exert a direct anti-inflammatory effect by inhibiting blood cell functions, such as platelet-leukocyte interactions and the exposure of cellular adhesion molecules, events recognized to play a crucial role in inflammatory responses. $^{30,31}$ We broadly confirm these hypotheses in our cohort of COVID-19-hospitalized patients.

Subgroup analyses in our study showed that heparin treatment was more effective in specific severe situations, such as in patients with a higher disease severity, requiring ICU admission, or with a strong coagulation activation as reported by D-dimer levels. The results of our study are in line with a retrospective analysis of 449 cases of severe COVID-19 pneumonia in China, which suggested first that use of LMWH for at least 7 days could lead to a survival advantage only in patients with higher levels of D-dimers or with a high composite score including laboratory and clinical parameters. ${ }^{6}$ Recently, Billett et al reported that at higher levels of D-dimer $(>10 \mu \mathrm{g} / \mathrm{mL}$ ), enoxaparin prophylaxis was associated with decreased mortality (odds ratio: 0.13, 95\% CI: 0.034-0.49), while UFH and enoxaparin therapies were not. $^{32}$ Similarly, in 786 patients (28\%) receiving systemic anticoagulation (including intravenous or subcutaneous UFH, LMWH, non-vitamin $\mathrm{K}$ antagonist oral ACs or, in few ICU cases, tissue plasminogen activator) during their hospital stay, a lower mortality was evident particularly in patients requiring mechanical ventilation. ${ }^{33}$

In late March 2020, the International Society on Thrombosis and Haemostasis recommended that all hospitalized COVID-19 patients, even those not in the ICU, should receive prophylactic doses of LMWH, unless with contraindications. ${ }^{18}$ After the publication of the first studies between the end of April and the beginning of May, the use of LMWH at prophylactic doses steeply increased. 6,33

In our hospitals, heparin was used in over $70 \%$ of cases (very largely as LMWH), in more than half of the cases at prophylactic doses. Heparin was used in the early phase after hospitalization, with or without previous laboratory evidence of coagulation activation at entry, thus supporting its use as a preventive therapy to potentially limit coagulation activation in subsequent phases. However, its efficacy appeared to be potentiated in subjects with evidence of hypercoagulation or with a more severe disease evolution, 
Table 3 Hazard ratios for mortality according to heparin use in different subgroups

\begin{tabular}{|c|c|c|c|}
\hline & Heparin NO $(N=770)$ & Heparin YES $(N=1,804)$ & \\
\hline Subgroups & No. death/patient at risk & No. death/patient at risk & $\mathrm{HR}(95 \% \mathrm{Cl})^{\mathrm{a}}$ \\
\hline \multicolumn{4}{|l|}{ Age } \\
\hline$<70 \mathrm{y}$ & $30 / 460$ & $51 / 942$ & $0.53(0.32-0.88)$ \\
\hline$\geq 70 y$ & $115 / 310$ & $202 / 862$ & $0.67(0.53-0.84)$ \\
\hline \multicolumn{4}{|l|}{ Gender } \\
\hline Women & $49 / 275$ & $95 / 714$ & $0.74(0.51-1.07)$ \\
\hline Men & $96 / 495$ & $158 / 1,090$ & $0.51(0.40-0.66)$ \\
\hline \multicolumn{4}{|c|}{ Highest degree of COVID-19 severity experienced at hospital } \\
\hline Mild pneumonia & $25 / 431$ & $33 / 790$ & $0.62(0.36-1.08)$ \\
\hline Severe pneumonia & $57 / 227$ & $72 / 509$ & $0.56(0.38-0.81)$ \\
\hline ARDS & $63 / 112$ & $148 / 505$ & $0.40(0.30-0.53)$ \\
\hline \multicolumn{4}{|l|}{ ICU } \\
\hline No & $118 / 663$ & $197 / 1,517$ & $0.63(0.50-0.80)$ \\
\hline Yes & $27 / 65$ & $53 / 262$ & $0.29(0.17-0.49)$ \\
\hline \multicolumn{4}{|c|}{ C-reactive protein at baseline ${ }^{\mathrm{b}}$} \\
\hline$<10 \mathrm{mg} / \mathrm{L}$ & $54 / 390$ & $60 / 597$ & $0.44(0.30-0.65)$ \\
\hline$\geq 10 \mathrm{mg} / \mathrm{L}$ & $86 / 332$ & $174 / 1,118$ & $0.68(0.53-0.88)$ \\
\hline \multicolumn{4}{|l|}{ CKD stage at baseline $^{c}$} \\
\hline Stage 1 & $21 / 286$ & $21 / 592$ & $0.26(0.14-0.49)$ \\
\hline Stage 2 & $47 / 278$ & $76 / 693$ & $0.61(0.42-0.89)$ \\
\hline Stage $3 a$ or stage $3 b$ & $51 / 135$ & $89 / 355$ & $0.81(0.56-1.16)$ \\
\hline Stage 4 or stage 5 & $20 / 37$ & $59 / 134$ & $0.66(0.42-1.05)$ \\
\hline \multicolumn{4}{|c|}{ Number of in-hospital therapies for COVID-19, including HCQ } \\
\hline 0 & $25 / 158$ & $36 / 131$ & $1.17(0.64-2.14)$ \\
\hline 1 & $48 / 236$ & $52 / 429$ & $0.58(0.39-0.87)$ \\
\hline 2 & $49 / 284$ & $95 / 709$ & $0.67(0.47-0.95)$ \\
\hline $3-5$ & $23 / 92$ & $70 / 535$ & $0.30(0.19-0.46)$ \\
\hline
\end{tabular}

Abbreviations: ARDS, acute respiratory distress syndrome; Cl, confidence intervals; CKD, chronic kidney disease; ICU, intensive care unit; $\mathrm{HCQ}$, hydroxychloroquine; HR, hazard ratios.

${ }^{a}$ Propensity score analysis, inverse probability weighting, including hospital index as a random effect covariate; multiple imputed analysis.

${ }^{b}$ Missing data for $N=137$. Data reported are based on a case complete analysis $(N=2,437)$ without missing for C-reactive protein. Multiple imputed analysis yielded very similar results.

${ }^{c}$ Missing data for $N=64$. Data reported are based on a case complete analysis $(N=2,510)$ without missing for eGFR. Multiple imputed analysis yielded very similar results.

confirming the importance of coagulation activation in the natural history of COVID-19.

A still open question on the use of anticoagulation in COVID-19 patients is if therapeutic doses of heparin are more effective than the low doses used in prophylaxis. In our study, both regimens were effective in reducing mortality, but the prophylactic doses at a higher extent (60 vs. $35 \%$ ). Probably the hemorrhagic potential of high doses of heparin, increased by the interaction with other drugs and comorbidities present in patients hospitalized with COVID-19, may partly overcome the potential benefit of full anticoagulation in these patients. On the other hand, we cannot exclude that the apparent benefit of prophylactic over therapeutic heparin could be that patients may have started on prophylaxis before they got a diagnosis of VTE qualifying them for therapeutic anticoagulation, hence the final contribution of therapy may have been diluted by prior prophylactic treatment. Also, indication bias is a potential reason why highdose heparin was associated with mortality at a lower extent in comparison with a prophylactic dose.

The major and well-known complications of heparin are bleeding and heparin-induced thrombocytopenia (HIT). Unluckily, we could not record information on adverse events. However, Pesavento et $\mathrm{al}^{34}$ showed in a smaller cohort of Italian patients that the rate of relevant bleeding was higher in patients treated with (sub)therapeutic doses of ACs, while overall mortality tended to be higher as compared with patients treated with standard prophylactic doses. Other observational studies reported that treatment with a therapeutic/higher dosage of AC drugs was associated with a higher incidence of bleeding, despite a decrease of inhospital mortality. ${ }^{7,35}$ HIT is a severe immune-mediated 
Table 4 Hazard ratios for mortality according to dosage of heparin

\begin{tabular}{|l|l|l|l|l|}
\hline \multicolumn{2}{|l|}{ Multiple imputation analysis $(N=\mathbf{2 , 5 7 4 )}$} & Patients at risk $(N=2,574)$ & Person-days & $\begin{array}{l}\text { Death rate }(x 1,000 \\
\text { person-days) }\end{array}$ \\
\hline \multicolumn{2}{|l|}{ Death $(N=398)$} & $770(100 \%)$ & 10,362 & 14.0 \\
\hline No, no. $(\%)$ & $145(18.8 \%)$ & $1,804(100 \%)$ & 34,301 & 7.4 \\
\hline Yes, no. (\%) & $253(14.0 \%)$ & $983(100 \%)$ & 18,857 & 6.1 \\
\hline Prophylactic regimen & $114(11.6 \%)$ & $418(100 \%)$ & 8,583 & 7.2 \\
\hline Therapeutic regimen & $62(14.8 \%)$ & $403(100 \%)$ & 6,861 & 11.2 \\
\hline Unknown (missing data) & $77(19.1 \%)$ & & $\mathrm{HR}(95 \% \mathrm{Cl})$ \\
\hline Hazard ratio for mortality (multivariable analysis $\left.{ }^{\mathrm{a}}\right)$ & & $0.40(0.30-0.52)$ \\
\hline Prophylactic doses vs. no heparin & & $0.65(0.46-0.93)$ \\
\hline Therapeutic doses vs. no heparin & & $1.54(1.06-2.25)$ \\
\hline Therapeutic vs. prophylactic regimen & & \\
\hline
\end{tabular}

Abbreviations: $\mathrm{Cl}$, confidence interval; $\mathrm{HR}$, hazard ratio.

${ }^{a}$ Controlling for age, sex, diabetes, hypertension, ischemic heart disease, chronic pulmonary disease, chronic kidney disease, C-reactive protein, HCQ, and other in-hospital therapies for COVID-19 as fixed effects and hospital index as a random effect.

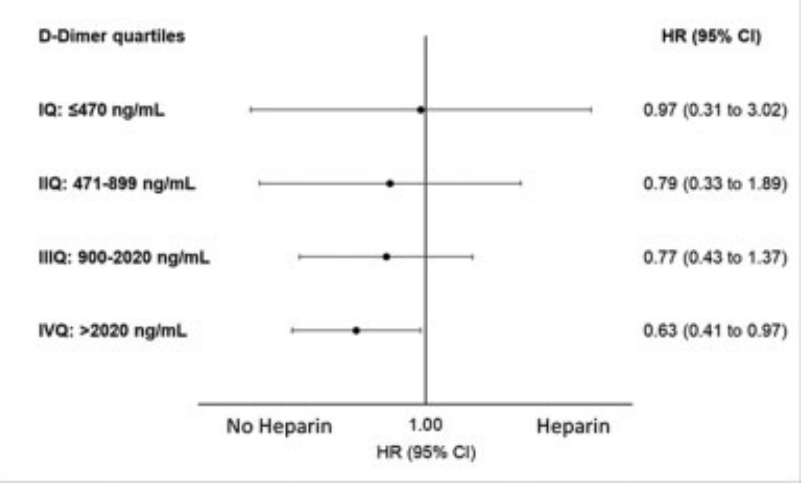

Fig. 2 Hazard ratios for mortality according to heparin use in D-dimer quartiles.

complication of heparin therapy, leading to platelet activation, platelet consumption, and thrombin generation. Paradoxically, HIT is characterized by both thrombocytopenia and a prothrombotic state. Recently, some reports presented the occurrence of HIT in COVID-19 patients. ${ }^{36,37}$ However, larger investigations are warranted to confirm this increased incidence of HIT and to understand its physiopathology. ${ }^{36}$

Our findings are similar to those of Ayerbe et al, showing that heparin treatment was associated with reduced mortality in 2,075 unselected patients with COVID-19 admitted to 17 hospitals in Spain. ${ }^{38}$ Additionally, focusing on LMWH therapy, recently Billett et $\mathrm{al}^{32}$ and Albani et $\mathrm{al}^{39}$ reported 51 and 50\%, respectively, lower risk of death in patients receiving prophylactic doses. In line with our findings, Albani et al found an inhospital mortality reduction of $46 \%$ at therapeutic regimen. ${ }^{39}$

\section{Strengths and limitations}

A major strength of this study is the large, unselected patient sample from 30 hospitals, covering the entire Italian territory.
Patient sampling covered all the overt epidemic period in Italy. Several statistical approaches were used to overcome biases due to the observational nature of the investigation.

This study has, however, several recognized limitations. First of all, we are well aware of the limits of a retrospective observational study. However, the CORIST Collaboration was launched at the very beginning of the pandemic, when the general situation in Italy was dramatic and the organization of a randomized controlled clinical trial was considered to be quite difficult. In the absence of any solid data, a prompt, real-life observational study appeared to be the best option at that moment. We took several precautions to account for the nonrandomized heparin administration procedure and to reduce the effects of confounders by using a propensity-score method.

Another limitation is that the study population is confined to Italy, and results obtained might not be applicable to other populations with possibly different geographical, racial and socio-economic conditions, and with possibly different natural history of COVID-19. Moreover, several parameters were not collected in all or some patients, and not all in-hospital medications might have been fully recorded. This is mainly due to our decision to interfere in a quite soft way with the dramatic clinical situation present in the majority of participating hospitals by proposing a relatively simple protocol, asking to report an essential dataset rather than a detailed, complete set of information; the latter could have distracted doctors from their therapeutic efforts and would have discouraged the participation of many colleagues. Information about regimen (prophylactic or therapeutic) was missing for $15.7 \%$ of the whole cohort. However, after the exclusion of patients for whom the type of heparin used was missing data, the association of heparin use with lower mortality was unchanged. A switch between regimens potentially occurred during hospitalization in some patients, but unfortunately this is missing information. For differing reasons, timing of the first dose of heparin after presentation to the hospital and duration of treatment could not be provided by some 
clinical centers. Time of heparin administration was not detailed; thus it was not possible to consider in Cox regression heparin as a time-dependent predictor. Additionally, specific reasons why patients were treated or not with heparin could not be collected; patients might have had contraindications or were considered to be inappropriate by physician's discretion. Adverse events possibly related to drug therapy also could not be documented.

Moreover, information about medication on admission was not collected and the chance that chronic therapy with ACs or platelet inhibitors before admission may be overrepresented in those COVID-19 patients who did not get (additional) prophylaxis should be considered.

Finally, the possibility of unmeasured residual confounders cannot be ruled out. However, the E-value for the lower boundary of the confidence interval for the protective association of heparin from death has the large value of 2.04 , indicating that the confidence interval could be moved to include the null only by a strong unmeasured confounder or combination of confounders associated with both heparin treatment and death, with a risk ratio of 2.04-fold, above and beyond all measured confounders. Weaker confounders could not do so.

\section{Conclusion}

Our study, including a large real-life sample of patients with COVID-19 hospitalized throughout Italy, shows that heparin use was associated with a $40 \%$ lower overall in-hospital mortality. In the absence of clear-cut results from randomized controlled trials, our data support the use of heparin in inpatients with COVID-19, particularly in severely ill patients.

Given the observational design of our study, however, these results should still be treated cautiously.

\section{What is known about this topic?}

- A hypercoagulable condition, associated with the presence of microvascular thrombosis, has been described in patients with COVID-19 and proposed as a possible pathogenic mechanism contributing to disease progression and lethality.

- Major guidelines supported the potentially beneficial effect of anticoagulation therapy, mainly low-molecular-weight heparin, in reducing mortality in COVID-19 patients. However, at present, evidence is insufficient.

\section{What does this paper add?}

- In a large real-life sample of patients with COVID-19 hospitalized throughout Italy, heparin use, mainly low-molecular-weight heparin, was associated with a $40 \%$ lower overall in-hospital mortality.

- In the absence of clear-cut results from controlled randomized trials, these findings support the use of heparin in patients with COVID-19, particularly in severely ill patients or in those with strong coagulation activation.

\section{Author Contributions}

Prof. Iacoviello and Di Castelnuovo had full access to all the data in the study and take responsibility for the integrity of the data and the accuracy of the data analysis. Concept and design: Di Castelnuovo, Costanzo, Iacoviello, and De Caterina. Acquisition, analysis, or interpretation of data: all authors. Drafting of the manuscript: Iacoviello, Di Castelnuovo and Costanzo. Critical revision of the manuscript for important intellectual content: Iacoviello, Di Castelnuovo, De Caterina, and de Gaetano Donati. Statistical analysis: Costanzo, Di Castelnuovo, and Arboretti. Administrative, technical, or material support: all authors. Supervision: Iacoviello and Di Castelnuovo.

\section{Funding}

None.

\section{Conflict of Interest}

None declared.

\section{Acknowledgments}

We thank the 30 participating clinical centers included in this cohort.

This article is dedicated to all patients who suffered or died, often in solitude, due to COVID-19; their tragic fate gave us moral strength to initiate and complete this research.

The authors alone are responsible for the views expressed in this article. They do not necessarily represent the views, decisions, or policies of the institutions with which they are affiliated.

\section{References}

1 Lippi G, Favaloro EJ. D-Dimer is associated with severity of coronavirus disease 2019: a pooled analysis. Thromb Haemost 2020;120(05):876-878

2 Tang N, Li D, Wang X, Sun Z. Abnormal coagulation parameters are associated with poor prognosis in patients with novel coronavirus pneumonia. J Thromb Haemost 2020;18(04):844-847

3 Llitjos JF, Leclerc M, Chochois C, et al. High incidence of venous thromboembolic events in anticoagulated severe COVID-19 patients. J Thromb Haemost 2020;18(07):1743-1746

4 Gazzaruso C, Paolozzi E, Valenti C, et al. Association between antithrombin and mortality in patients with COVID-19. A possible link with obesity. Nutr Metab Cardiovasc Dis 2020;30(11): 1914-1919

5 Marongiu F, Grandone E, Barcellona D. Pulmonary thrombosis in 2019-nCoV pneumonia? JThromb Haemost 2020;18(06):1511-1513

6 Tang N, Bai H, Chen X, Gong J, Li D, Sun Z. Anticoagulant treatment is associated with decreased mortality in severe coronavirus disease 2019 patients with coagulopathy. J Thromb Haemost 2020;18(05):1094-1099

7 Nadkarni GN, Lala A, Bagiella E, et al. Anticoagulation, bleeding, mortality, and pathology in hospitalized patients with COVID-19. J Am Coll Cardiol 2020;76(16):1815-1826

8 Tremblay D, van Gerwen M, Alsen M, et al. Impact of anticoagulation prior to COVID-19 infection: a propensity scorematched cohort study. Blood 2020;136(01):144-147

9 Wang T, Chen R, Liu C, et al. Attention should be paid to venous thromboembolism prophylaxis in the management of COVID-19. Lancet Haematol 2020;7(05):e362-e363 
10 Danzi GB, Loffi M, Galeazzi G, Gherbesi E. Acute pulmonary embolism and COVID-19 pneumonia: a random association? Eur Heart J 2020;41(19):1858

11 Rotzinger DC, Beigelman-Aubry C, von Garnier C, Qanadli SD. Pulmonary embolism in patients with COVID-19: time to change the paradigm of computed tomography. Thromb Res 2020; 190:58-59

12 Cattaneo M, Morici N. Is thromboprophylaxis with high-dose enoxaparin really necessary for COVID-19 patients? A new "prudent” randomised clinical trial. Blood Transfus 2020;18(03): 237-238

13 Cattaneo M, Bertinato EM, Birocchi S, et al. Pulmonary embolism or pulmonary thrombosis in COVID-19? Is the recommendation to use high-dose heparin for thromboprophylaxis justified? Thromb Haemost 2020;120(08):1230-1232

14 World Health Organization. Clinical management of severe acute respiratory infection (SARI) when COVID-19 disease is suspected. WHO/2019-nCoV/clinical/2020.4 2020. Accessed February 1, 2021 at: https://www.who.int/docs/default-source/coronaviruse/clinical-management-of-novel-cov.pdf

15 CDC. Interim clinical guidance for management of patients with confirmed coronavirus disease (COVID-19). Published 2020. Updated April 13, 2020. Accessed September10, 2020 at: https://www.cdc.gov/coronavirus/2019-ncov/hcp/clinical-guidance-management-patients.html

16 Matos R, Chung K. DoD COVID-19 practice management guide: clinical management of COVID-19. DoD. Published 2020. Updated March 23, 2020. Accessed September 10, 2020 at: https://asprtracie.hhs.gov/technical-resources/resource/7899/dod-covid-19practice-management-guide-clinical-management-of-covid-19

17 Marietta M, Ageno W, Artoni A, et al. COVID-19 and haemostasis: a position paper from Italian Society on Thrombosis and Haemostasis (SISET). Blood Transfus 2020;18(03):167-169

18 Bikdeli B, Madhavan MV, Jimenez D, et al; Global COVID-19 Thrombosis Collaborative Group, Endorsed by the ISTH, NATF, ESVM, and the IUA, Supported by the ESC Working Group on Pulmonary Circulation and Right Ventricular Function. COVID-19 and thrombotic or thromboembolic disease: implications for prevention, antithrombotic therapy, and follow-up: JACC Stateof-the-Art Review. J Am Coll Cardiol 2020;75(23):2950-2973

19 Thachil J, Tang N, Gando S, et al. ISTH interim guidance on recognition and management of coagulopathy in COVID-19. J Thromb Haemost 2020;18(05):1023-1026

20 Gerotziafas GT, Catalano M, Colgan MP, et al; Scientific Reviewer Committee. Guidance for the management of patients with vascular disease or cardiovascular risk factors and COVID-19: position paper from VAS-European Independent Foundation in Angiology/Vascular Medicine. Thromb Haemost 2020;120(12): 1597-1628

21 Di Castelnuovo A, De Caterina R, de Gaetano G, Iacoviello L. Controversial relationship between renin-angiotensin system inhibitors and severity of COVID-19: announcing a large multicentre case-control study in Italy. Hypertension 2020;76(02): 312-313

22 Di Castelnuovo A, Bonaccio M, Costanzo S, et al. Common cardiovascular risk factors and in-hospital mortality in 3,894 patients with COVID-19: survival analysis and machine learning-based findings from the multicentre Italian CORIST Study. Nutr Metab Cardiovasc Dis 2020;30(11):1899-1913
23 Di Castelnuovo A, Costanzo S, Antinori A, et al. Use of hydroxychloroquine in hospitalised COVID-19 patients is associated with reduced mortality: Findings from the observational multicentre Italian CORIST study. Eur J Intern Med 2020;82:38-47

24 WHO. Clinical management of severe acute respiratory infection when novel coronavirus (2019-nCoV) infection is suspected: interim guidance, 28 January 2020. Accessed September 10, 2020 at: https://apps.who.int/iris/bitstream/handle/10665/330893/WHOnCoV-Clinical-2020.3-eng.pdf? sequence $=1$ \&isAllowed $=y$

25 Rubin DB. Multiple Imputation for Nonresponse in Surveys. New York, NY: John Wiley; 1987

26 Glidden DV, Vittinghoff E. Modelling clustered survival data from multicentre clinical trials. Stat Med 2004;23(03):369-388

27 Garrido MM, Kelley AS, Paris J, et al. Methods for constructing and assessing propensity scores. Health Serv Res 2014;49(05):1701-1720

28 VanderWeele TJ, Ding P. Sensitivity analysis in observational research: introducing the E-value. Ann Intern Med 2017;167 (04):268-274

29 Di Gennaro F, Marotta C, Storto M, et al. SARS-CoV-2 transmission and outcome in neuro-rehabilitation patients hospitalized at neuroscience hospital in Italy. Mediterr J Hematol Infect Dis 2020;12(01):e2020063

30 Maugeri N, de Gaetano G, Barbanti M, Donati MB, Cerletti C. Prevention of platelet-polymorphonuclear leukocyte interactions: new clues to the antithrombotic properties of parnaparin, a low molecular weight heparin. Haematologica 2005;90(06):833-839

31 Maugeri N, Di Fabio G, Barbanti M, de Gaetano G, Donati MB, Cerletti C. Parnaparin, a low-molecular-weight heparin, prevents P-selectin-dependent formation of platelet-leukocyte aggregates in human whole blood. Thromb Haemost 2007;97(06):965-973

32 Billett HH, Reyes-Gil M, Szymanski J, et al. Anticoagulation in COVID-19: effect of enoxaparin, heparin, and apixaban on mortality. Thromb Haemost 2020;120(12):1691-1699

33 Paranjpe I, Fuster V, Lala A, et al. Association of treatment dose anticoagulation with in-hospital survival among hospitalized patients with COVID-19. J Am Coll Cardiol 2020;76(01):122-124

34 Pesavento R, Ceccato D, Pasquetto G, et al. The hazard of (sub) therapeutic doses of anticoagulants in non-critically ill patients with Covid-19: The Padua province experience. J Thromb Haemost 2020;18(10):2629-2635

35 Ionescu F, Jaiyesimi I, Petrescu I, et al. Association of anticoagulation dose and survival in hospitalized COVID-19 patients: a retrospective propensity score-weighted analysis. Eur J Haematol 2020;106(02):165-174

36 Bidar F, Hékimian G, Martin-Toutain I, Lebreton G, Combes A, Frère C. Heparin-induced thrombocytopenia in COVID-19 patients with severe acute respiratory distress syndrome requiring extracorporeal membrane oxygenation: two case reports. J Artif Organs 2021;24(2):277-281

37 Lingamaneni P, Gonakoti S, Moturi K, Vohra I, Zia M. Heparininduced thrombocytopenia in COVID-19. J Investig Med High Impact Case Rep 2021;13(1):8857

38 Ayerbe L, Risco C, Ayis S. The association between treatment with heparin and survival in patients with Covid-19. J Thromb Thrombolysis 2020;50(02):298-301

39 Albani F, Sepe L, Fusina F, et al. Thromboprophylaxis with enoxaparin is associated with a lower death rate in patients hospitalized with SARS-CoV-2 infection. A cohort study. EClinicalMedicine 2020;27:100562 